\title{
La ciudad y el espacio público: la diversidad fragmentada*
}

\author{
The city and the public space: \\ fragmented diversity
}

\author{
CLAUDIA TERESA GASCA MORENO** \\ AQUILES OMAR ÁVILA QUIJAS***
}

\begin{abstract}
This paper scrutinizes the way diversity is understood in urban zones and cities. It seeks to demonstrate that diversity is a consequence of the fragmentation of social interactions drawn from the blurring of the other in order to root out liberal subject, the one that holds political and social equality. In this sense, we propose looking once again at the city and public space from the perspective of its distances and social separation, as well as the recognition of social absences in what is called "plurality" that, nowadays, demands an interdisciplinary dialogue and creatively crafted methodological frames.
\end{abstract}

Key words: uses, appropriation, urban realm, fragmentation

\begin{abstract}
Resumen
Este trabajo pone a discusión la manera como se entiende la diversidad en las zonas urbanas y en las ciudades. Parte de la idea de que esta diversidad en realidad es consecuencia de una fragmentación de las interacciones sociales que se dan a partir de la difuminación de la alteridad en pos del seguimiento del individuo liberal, es decir, aquel que es igual política y socialmente. En este sentido, proponemos volver a mirar a la ciudad y el espacio público desde sus separaciones y distancias sociales, reconocer las ausencias en la pluralidad contenida y que hoy por hoy nos demanda indagardesde un diálogo interdisciplinario y propuestas metodológicas creativas.
\end{abstract}

Palabras clave: usos, apropiación, ámbito urbano, fragmentación

\section{Introducción}

T a tradición analítica en la historia y la ciencia política parte de la idea de que lo urbano y lo relacionado Lcon la ciudad contiene lo diverso, es decir, hablar de ciudad significa hacerlo de una multiplicidad de realidades que se yuxtaponen, entrecruzan, sobreponen las unas con las otras para formar un galimatías que sólo adquiere orden con la categorización de zonas, lo que permite hacer un dibujo mental y, sobre todo, lineal del espacio público que lo ordena tanto material como culturalmente, de tal suerte que se generan fronteras entre los distintos actores y grupos sociales que lo comparten, por lo que la visión antropológica es necesaria.

* Artículo recibido el 20/08/19 y aceptado el 20/12/19.

** Universidad de Guanajuato-Campus León, Departamento de Estudios Sociales, Blvd. Puente Milenio 1001, Fracción del Predio de San Carlos, 37670, León, Gto. <ct.gasca@ugto.mx> orciD: https: / /orcid.org/0000-0002-7477-1698.

*** Universidad de Guanajuato, Campus León-Departamento de Estudios Sociales, Blvd. Puente Milenio 1001, Fracción del Predio de San Carlos, 37670, León, Gto. <avilaquijas@ugto.mx> orcid: https://orcid.org/0000-0003-1040-8037. 
Considerado de esa manera, hay un ordenamiento preciso a partir de la composición semántica del espacio, lo que marca y determina los usos y apropiaciones del mismo. Sin embargo, ¿son estas categorías las que determinan la diversidad? ¿Qué es lo que podemos ver si cambiamos el prisma con el que hemos observado hasta ahora la ciudad y los fenómenos urbanos? ¿Cuáles son los elementos que nos permitirían discernir las diversidades que se contienen en los espacios organizados en campos semánticos? ¿Cómo desinvisibilizar el conjunto de elementos del entorno que dibujan lo multifacético? La tradición analítica de la antropología urbana puede ofrecer algunas pistas al respecto. En consecuencia, lo que este texto presentará es el análisis del espacio público como el crisol que hemos construido para albergar la diversidad y que, no obstante, en la realidad parece ser un recipiente de exclusiones e invisibilizaciones de subconjuntos de la sociedad. Si partimos de la idea de que la diversidad es una construcción social, que reconoce la existencia del otro en cuanto es diferente al marco referencial a partir del cual se construye al sujeto liberal que es idealizado y, por ende, homogéneo (Guédez, 2005; Banerjee y Duflo, 2019), ¿cómo observar con nuevos lentes una realidad que parece más compleja de lo que fue establecido?

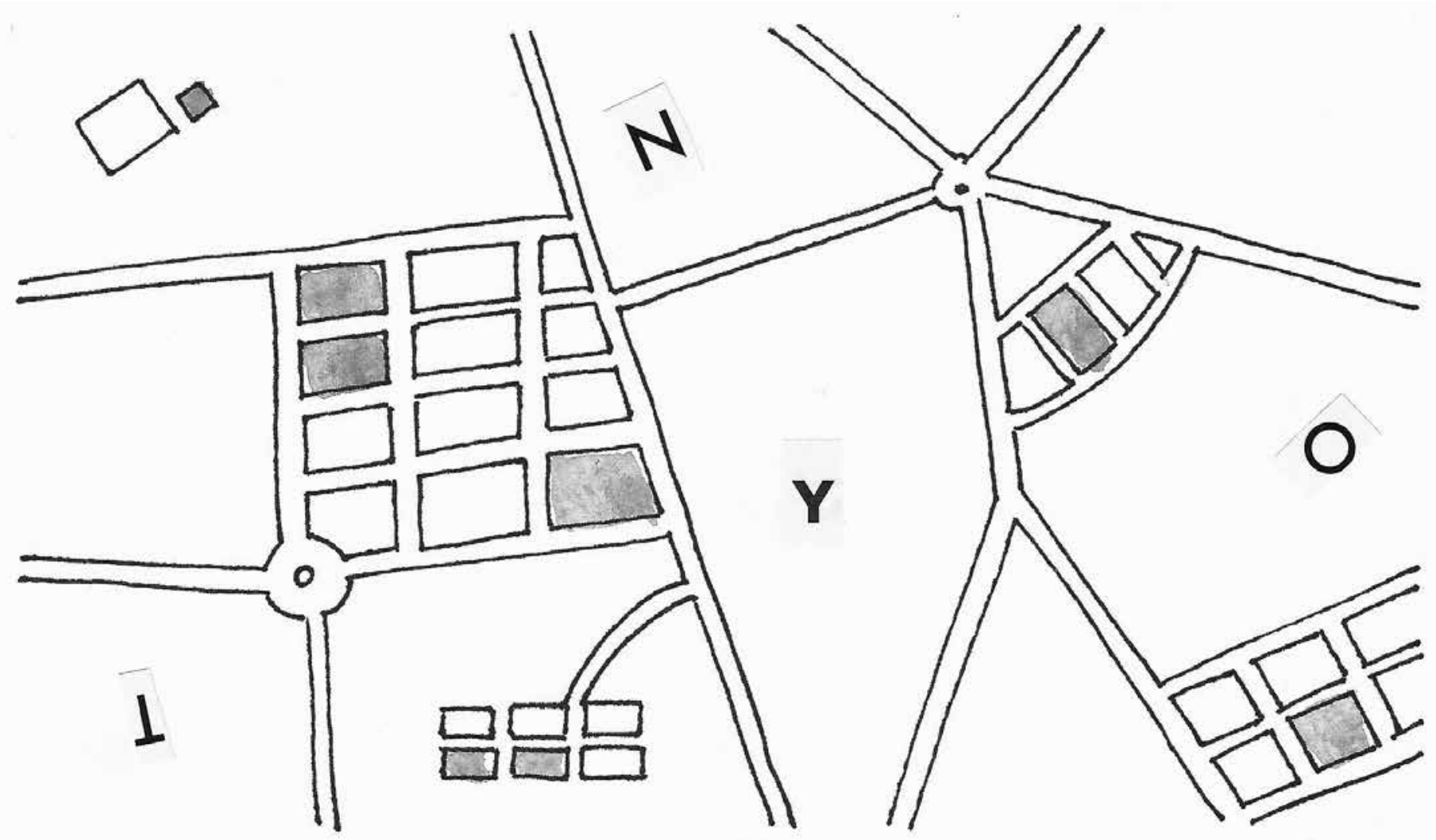

\section{El espacio público en primer plano}

El estudio del espacio público como punto de partida para la investigación de la diversidad en las ciudades no es algo nuevo, en la década de los setenta y principios de los ochenta el concepto adquirió relevancia en disciplinas como arquitectura, sociología, geografía, antropología, entre otras, que tenían como objetivo el estudio de la dinámica en la urbe, esta noción se convirtió en un referente de lo diverso y de múltiples posibilidades y aristas desde donde podría abordarse el estudio de la ciudad (Portal, 2007: 57).

A mediados del siglo xix la noción de lo público desde la experiencia urbana se relacionó con una materialidad territorial y un uso colectivo que debía garantizar el Estado, pero que el modelo económico capitalista y su consolidación ponía en crisis con la defensa de lo privado (Portal, 2007: 58). Esta tendencia produjo nuevas interrelaciones en la experiencia urbana, donde incluso llegó a cuestionarse la existencia del espacio público y su condición de "accesible para todos". Este desvanecimiento tuvo repercusiones en el ámbito académico, donde el concepto espacio público se usó sin distinción para referirse a sitios de acceso abierto, pero también para aludir a lugares del ámbito privado como los supermercados o plazas comerciales. 
En el abordaje de la experiencia de la ciudad y sus espacios, lo público se ha definido a partir del uso colectivo y de una aparente heterogeneidad de usuarios que se apropian de él en función de sus intereses, imaginarios y percepciones. Estos usos son impuestos por actores privilegiados que establecen cómo y cuándo deben usarse (Portal, 2007: 58) y, aunque existen resignificaciones y apropiaciones que se oponen al uso original para el que fueron creados, hay una regulación permanente que sin duda acota la experiencia de los habitantes en la urbe. Hasta hace poco más de una década, la escritura antropológica sobre el espacio público y la ciudad acentuaba la importancia de este binomio como una estrategia para aproximarse a la dinámica de escenarios de un tipo insólito, organizados en torno al anonimato y compuestos de relaciones efímeras que ponían en el centro de la discusión la diversidad, la heterogeneidad y la diferencia de la vida urbana (Vergara, 2005; Licona, 2007a y 2007b; Portal, 2007; Delgado, 2008). Estas etnografías revelaron al espacio público como organizador de la vida en las ciudades y detonante de lo diverso por, aparentemente, aglutinar prácticas heterogéneas y expresarse de forma simultánea por distintos tipos de actores como transeúntes, turistas, amantes, trabajadores, comerciantes, vagabundos, por mencionar algunos.

Abilio Vergara (2005: 7) señala cuatro momentos del espacio público: a) sus funciones se reducían al ritual-político o ritual-religioso; el espacio era productor de actores, pueblo y dirigencia, al mismo tiempo que arena de contienda; después, $b$ ) adquirió características de foro para escenificar lo identitario; enseguida, c) la relación producción-consumo configuró relaciones entre la generación de un público y clientes que se materializaron espacialmente; por último, d) pasó a ser un escenario de recreación y convivencia. Nosotros agregaríamos un momento más, el espacio público en cuanto escenario de la exclusión y la segregación en la ciudad contemporánea.

Las calles, las plazas, los jardines o los mercados -todos éstos, espacios públicos identificables en las ciudades- han sido explorados como sitios donde el antropólogo urbano podía presenciar la actividad social "al natural", sin obstaculizarla o interferir en ella (Delgado, 2008: 13). En su momento fue revelador concebir al espacio público como una entrada a la alteridad flotante (Delgado, 2008: 26) porque a primera vista parecía agrupar todo lo contrario a lo homogéneo y cobijado en un territorio determinado. Las exploraciones manifestaban las prácticas y expresiones de usuarios multifacéticos, poblaciones en tránsito continuo, actores que sólo se atrincheraban en horarios específicos o bajo ciertas circunstancias, usos y apropiaciones provisionales e intermitentes, que nos hicieron pensar el espacio público como estandarte de lo diverso, lo que planteó, sin duda, un conjunto de retos teóricos y metodológicos para la antropología urbana.

Todos estos trabajos ya advertían un punto neurálgico que Manuel Delgado (2008) resume en la pregunta: ¿quién tiene derecho a usar los espacios públicos? Cuestionamiento que pone en tela de juicio una de sus características más laureadas: la diversidad. En estos párrafos iniciales hemos insistido en que la heterogeneidad del espacio público es aparente, una suerte de ficción, en la medida en que aglutina diversidades "compatibles" con puntos de confluencia cuyos conflictos suelen ser negociables o detonan la puesta en marcha de estrategias para compartir y permanecer en el espacio en cuestión. En caso de que los usos, usuarios y prácticas resulten discordantes, ¿cuáles son las reacciones? ¿Cuáles ejercicios a partir de los que se resuelve un conflicto simbólico cuyo escenario es el espacio, en principio, concebido y construido para todos? Lo que nosotros observamos es un conjunto de desigualdades en el acceso al espacio urbano, que detona procesos de expulsión y segregación del grupo menos favorecido o con menos recursos para permanecer en él, y además propicia alejamientos de actores que hemos estudiado desde otros enfoques y temáticas, pero que podrían estar ausentes de nuestra idea de diversidad del espacio público urbano.

En México encontramos numerosos trabajos que analizan las dinámicas del espacio público desde su variedad de prácticas y relaciones, investigaciones que han abordado las nuevas configuraciones de lo público y lo privado (Rojas, 2007), relaciones entre el espacio público y el comercio (Monnet, 1996), la privatización y las formas de autosegregación residencial (Giglia, 2002). Una de las vetas más ricas en el estudio de las ciudades modernas ha sido la referente a los jóvenes y el espacio público urbano. Diversos investigadores del fenómeno juvenil (Valenzuela, 1988; Urteaga CastroPozo y Cornejo Portugal, 1995; Urteaga Castro-Pozo, 2007) han privilegiado el análisis del uso del tiempo libre en las ciudades proyectando a los jóvenes como los principales actores sociales del espacio público (Urteaga Castro-Urteaga, 2007: 82). También destacan los trabajos de Mauricio List Reyes sobre los usos a partir de la condición de género y preferencia sexual (List, 2007a: 193; y 2007b: 120). Trabajos más recientes nos llevan a reflexionar en el espacio público como expresión de lo global (De la Peña, 2015), como ente patrimonial (Del Mármol, Frigolé y Narotzky, 2010) y perímetro de conflicto (Almada, 2014) hasta aterrizar en los procesos de revitalización y puesta en venta de 
las ciudades (Giglia, 2018; Téllez, 2018). En conjunto, ponen de manifiesto que la ciudad se configura como un mosaico de diversidades que el espacio público fragmenta porque únicamente permite la convivencia entre iguales o similares, lejos de aquella idea que por décadas abrigó nuestras reflexiones sobre la ciudad y sus espacios. En otras palabras, en la apropiación del espacio público se detonan conflictos inmateriales y materiales cuyo corolario es la construcción de islotes sociales integrados por aquellos que cumplen con el discurso institucional o, bien, con el esquema informal de uso del sitio. De tener la razón, esto lleva a proyectar nuevas formas de observar la ciudad y los fenómenos urbanos, no como un monolito, sino desde su fragmentación. Concebir el espacio público como elemento restrictivo de la diversidad tiene implicaciones teóricas y metodológicas que nos obligan a desinvisibilizar nuestros supuestos, casi idealistas, del espacio público y la diversidad contenida en ellos, se trata de reconocer las limitantes y plantear nuevas estrategias para el abordaje de las múltiples diversidades fragmentadas. ¿Cómo se llegó a este punto? ¿Por qué pensamos esto?

\section{La diversidad difuminada El proyecto liberal y sus consecuencias}

Para el mundo occidental el cuestionamiento a las monarquías absolutas partió de la escasa o nula representación política que los diversos grupos integrantes de aquellas sociedades tenían en los órganos de toma de decisiones. Es decir, la invisibilización de la diversidad fue el motor a partir del cual se evolucionó a una forma de organización que la visibilizó, reconoció y, en última instancia, volvió a invisibilizarla.

La gestación de la igualdad política, primero entre los hombres, para luego y poco a poco, incluir a las mujeres, asumió que todos los individuos merecían ser representados a través de la obtención de la ciudadanía. Es decir, que todos tuvieran los mismos derechos sólo era posible a partir del cumplimiento de algunos requisitos. Si se cumplían se accedía a la posibilidad de incidir en la toma de decisiones; pero se eliminaba la diversidad al aceptar la calidad ciudadana, que no es otra cosa más que la homogeneización del individuo con el fin de construir un ciudadano prototípico, adecuado para un proyecto específico de nación. El triunfo del liberalismo es, también, la validación política de la anulación de la diversidad.

En la actualidad, con la evolución del concepto democracia, que va de la mano con el desarrollo liberal, la diversidad es reconocida, incluso, como parte de la riqueza cultural de una sociedad. También, como ya se dijo, se ha establecido que las ciudades, casi por definición, son contenedores de lo diverso. ¿Cómo encontrar un punto de equilibrio entre el principio liberal de igualdad política y social y la diversidad? El desarrollo político del mundo occidental no lo tiene en la agenda, por el contrario, como lo plantea Pierre Rosanvallon, nos hallamos ante el surgimiento de una sociedad de lo particular. Es decir, los vínculos sociales se gestan ya no en torno a estructuras macro de lo identitario, sino a "acoplamientos selectivos, acercamientos puntuales, caminos paralelos" (Rosanvallon, 2010: 100). Esto es, los proyectos que conformaron lo que hemos denominado Estados-nacionales pasaron por el tamiz de la generación de un conjunto de símbolos identitarios que permitieron la cohesión social en torno a abstracciones como nación, patria, lo mexicano (en el caso de nuestro país), y con ello configuraron un individuo ideal que se convertiría en la representación aspiracional de una sociedad que, en ese punto, había cedido su alteridad en pos de formar parte de algo que lo superaba como individuo. La idealización del sujeto es resultado del desarrollo histórico-social de cada población y, por supuesto, cambia a lo largo del tiempo (Lacarrieu, 2007), lo que plantea Rosanvallon es que la cohesión social hoy ya no depende de esas formas abstractas, sino de mecanismos que están asociados más con la interacción entre aquellos que comparten intereses comunes y establecen puntos simbólicos y físicos de encuentro, hay una selección del par que supera a la patria y a la nación, la reproducción de lo mexicano ya no está en la agenda que define los acercamientos, encuentros y sociabilidades. ¿Cómo define esto el uso del espacio público?

Hasta hoy en día la agenda de investigación sobre la gobernanza del espacio público ha privilegiado el análisis de las grandes ciudades, por ejemplo Mier y Terán, Vázquez y Ziccardi (2012) para la Ciudad de México; Hernández García (2013) para Bogotá. Lo que podemos plantear como hipótesis es que, en consonancia con aquella sociedad de lo particular, los gobiernos no niegan la diversidad; sin embargo, no están interesados en gestionar espacios públicos en los que se manifieste todo lo que es diferente a su idea de ciudadano. Por ello, el ejercicio administrativo ha tendido a la zonificación del espacio, a la construcción de campos semánticos sobre el mismo (Melé, 2016). La creación de zonas implica organizar el territorio, pero también a la sociedad, pues se establecen límites para las prácticas sociales en el territorio: "aquí no se puede..."; "eso puede hacerse allá"; "aquí se permite...". Los campos semánticos actúan de igual manera sólo que en lugar de organizar físicamente el 
espacio lo hacen simbólicamente, de tal suerte que los territorios generan fronteras invisibles y formas de actuar que se corresponden con el espacio en el que se está, comparte, cohabita. En el fondo, lo que sucede es que hay un proceso de exclusión velada que privilegia, por un lado, al ciudadano modelo, es decir, a aquel que representa la igualdad política y social del proyecto liberal y, por otro, fragmenta la diversidad al generar ínsulas de convivencia entre los diferentes grupos sociales que comparten y cohabitan un mismo espacio público. En nuestro ejercicio etnográfico se puede, incluso, observar una autosegregación que es el culmen del éxito de la exclusión y fragmentación de lo diverso. El individuo es consciente de que no empata con el modelo de ciudadano y opta por mantenerse al margen, según conciba el uso del espacio: “¿qué voy a hacer allá?”; "esos lugares no son para gente como nosotros". De tal suerte que hay un tipo de usuario definido por la zonificación o por el campo semántico que empata con el discurso de la diversidad y permite validar la invisibilización de los marginales, no obstante ser objeto de una exclusión que se produce a partir de la fragmentación de las sociabilidades, de la particularización de éstas.

Esta paradoja inherente: incluir-excluir, a la vez, promueve un conjunto de resistencias que obligan a poner el espacio público como un sujeto de negociación. La negociabilidad del espacio público se da a partir de la interacción de los ordenamientos formales e informales que se tejen sobre un territorio zonificado o delimitado simbólicamente (Giglia, 2016: 385).

Todos los espacios públicos, de manera general, en algunos casos de modo particular, tienen un ordenamiento normativo, reglas formales que deben ser observadas por cada uno de los usuarios y que suponen, en el caso de no hacerlo, sanciones de diferente tipo, aunque sobresalen las sociales. A pesar de esto, también se gestan reglas informales que en muchas ocasiones delimitan zonas y campos semánticos, lo que promueve un proceso constante de negociación entre autoridades y usuarios, y entre los mismos usuarios (Azuela, 2016: 15). De acuerdo con el planteamiento neoinstitucional, lo que se busca es generar marcos de certidumbre que sean garantes de las relaciones sociales, aunque lo que éstas generen sean procesos de exclusión y fragmentación de la diversidad.

Bajo esta lógica, ¿hemos de entender el territorio como un espacio en constante disputa? ¿Como un anclaje que marca y determina la identificación colectiva, como nos dice la antropología clásica que sucede? (Hoffman y Morales, 2018). Desde esta perspectiva, el territorio no es un recurso en disputa, pues las acciones sociales no se entienden a partir de su posi- cionamiento territorial, sino de su lugar, existente o inexistente, en el espacio público. La presencia y la no presencia tienen cosas que decir para el etnógrafo y para el análisis de las ciudades, con su respeto y promoción de la diversidad. El territorio deja de ser un espacio que conceptualiza las interacciones, son las interacciones las que le dan sentido al espacio y al territorio. Esto representa un reto metodológico, ¿cómo observar una realidad que es más compleja de lo que fue establecido? ¿Cómo aproximarnos a esos fragmentos y desde dónde hacerlo?

\section{Nuevos lentes, nuevos problemas: la diversidad fragmentada como reto metodológico}

Explorar las particularidades de la diversidad contemporánea en las ciudades desde una mirada renovada del espacio público que haga posible la distinción y análisis de las pluralidades fragmentadas requiere un giro metodológico acorde a la posición o, mejor dicho, cuestionamiento que planteamos. Desde hace más de una década, la experiencia de la antropología urbana puso en relieve la importancia del intercambio interdisciplinario en el estudio de la diversidad expresada en torno a temáticas como el patrimonio, el consumo cultural, los imaginarios juveniles y las fiestas en las ciudades (García Canclini, 2005; Rosas Mantecón, 2005; Nivón, 2005; Urteaga Castro-Pozo y Feixa, 2005; Sevilla y Portal, 2005). También reveló el dinamismo de los espacios estudiados, así como una variedad de actores, prácticas, relaciones, estilos de vida y otras expresiones que fueron abarcables en términos de la escala analizada. No obstante, hay intersecciones que no han sido agotadas y que plantean claroscuros en el abordaje y comprensión de la diversidad, justo ahí es donde identificamos múltiples posibilidades de análisis y comprensión de lo que pudo haber quedado sin rastrear (Balbi, 2017: 11).

Aunque la dinámica de las ciudades ha obligado al antropólogo urbano a modificar la duración del trabajo de campo, el tipo de vínculos y a incorporar en su pesquisa nuevas tecnologías para aproximarse a las interacciones y configuración de espacialidades, como es el caso de las redes sociales o los activismos que se gestan en la virtualidad, cuyas dinámicas e incidencia en el ámbito social y cultural eran inimaginables hasta hace un par de décadas, también lo ha conducido por un camino donde la creatividad metodológica y el diálogo interdisciplinario son imperiosos para comprender la actuación de los urbanitas. Si partimos de la idea de que el espacio público está fragmentado en 
un conjunto de ínsulas homogéneas donde se expresan prácticas, interacciones, relaciones, imaginarios y estilos de vida de los habitantes de las ciudades que no se tocan entre sí, al contrario, se repelen, la antropología ofrece la lupa para mirar minuciosamente lo que ocurre en ellas, mientras que disciplinas como la geografía, el urbanismo o la historia, entre otras, proponen un conjunto de lentes para amplificar, ensamblar, relacionar, comparar o incluso distanciarse para obtener una mirada de conjunto. Es necesaria una suerte de eclecticismo metodológico y estratégico (Durán Segura, 2011 ; Restrepo, 2012) para iluminar esos claroscuros alojados en lo cotidiano e inestable.

El espacio público es una fórmula para aproximarnos a lo urbano, ocurren negociaciones colectivas, adquieren visibilidad mientras que otras se diluyen en el anonimato (Delgado, 2008) por tal razón es importante reflexionar sobre la forma en que nos aproximamos a esas realidades y los lentes que utilizamos para ello. En ese sentido, es sustancial repensar el abordaje de la ciudad y sus espacios desde formas radicales, es decir, desde experiencias no normadas, encuentros azarosos y a la deriva (Durán Segura, 2011: 138); mirar y describir lo que ocurre en el espacio público a partir de un ejercicio de contemplación que nos permita identificar las expresiones y negociaciones de la diversidad y la manera como es fragmentada.

Para lograr una actitud crítica frente a lo que ocurre ahí, en el marco de las dinámicas contemporáneas, requerimos echar mano de una pluralidad de medios para obtener información, desarrollar un ojo reflexivo hacia cómo se practica el espacio, cómo se concibe (campos semánticos), pero al mismo tiempo cómo se fragmenta. Estamos frente a pequeñas piezas que hay que analizar con medios y herramientas que rayan en lo intuitivo, pero son la única vía de intentar su comprensión. Si en el espacio público hay una socialización en constante cambio ¿cómo nos aproximamos a sus dinámicas si no es que en la misma lógica? Un ejemplo de esto lo plantea Leal Sorcia (2012: 112) en su estudio de los migrantes indígenas y sus dinámicas en el espacio público urbano. Esta autora identifica que la interacción de grupos étnicos con la ciudad revela contextos interactivos de oscilación cultural que se manifiestan a través de nuevas prácticas en los parques, calles, mercados, comercios y otros espacios. $\mathrm{El}$ análisis de las interrelaciones de estos grupos no

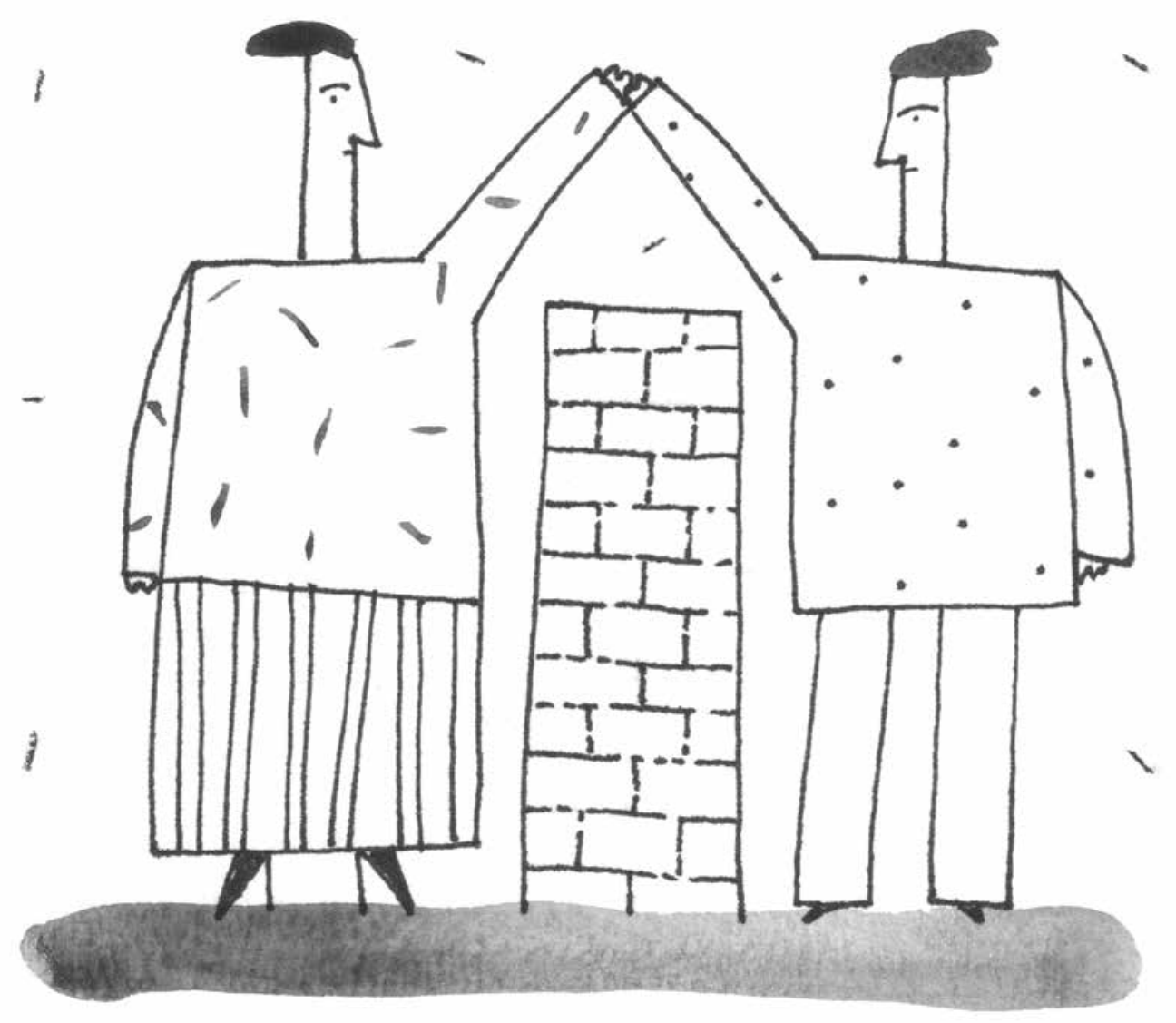


se enfoca (como por tradición se ha tratado en la bibliografía antropológica) exclusivamente en el ámbito sociocultural (Chávez, 2014; Leal, 2012), sino en los espacios donde se construyen interpretaciones y se interioriza la vida cotidiana mediante desplazamientos, inserciones y relaciones desde la continuidad e interdependencia con la dinámica urbana, por ejemplo en la escuela o la celebración de fiestas, entre otras.

Una estrategia útil para estudiar el espacio público y estos fragmentos es hacerlo desde los procesos de inserción de los actores, como lo sugiere Leal (2012) para los grupos étnicos en la ciudad, y que funciona para el análisis de colectividades urbanas que pueden o no estar visibles en lo público, para lograrlo es necesario realizar etnografías con visitas cortas pero intensas, combinar escritura y grabaciones de audio y video, enfocar la mirada en aspectos muy concretos que nos permitan hacer más eficientes los tiempos de investigación (Montes de Oca Barrera, 2015: 28). Las etnografías enfocadas nos ayudan a recuperar las muestras de diversidad que los espacios públicos contienen en fracciones y que son constantemente reordenadas por el ritmo de vida urbano. No hay otro modo de aproximarnos a estos elementos que hacerlo por partes, porque así están dispuestos en la vida social. Si la mirada tiene que posarse en lo cotidiano, en lo sutil y en lo minúsculo del espacio público (Durán Segura, 2011: 138), pero sólo tenemos acceso a distintas partes que pueden o no estar conectadas entre ellas, tenemos que emplear estrategias como las etnografías colectivas para intentar reunir las piezas del rompecabezas, combinarlas con miradas a diversas escalas, considerar experiencias etnográficas encorporadas en las cuales nuestros cuerpos también operen como vehículo para acompañar y entender lo que ocurre en los espacios urbanos y las prácticas de grupos que se nos escurren de la dinámica urbana (Jirón y Walter, 2016).

La idea de una diversidad fragmentaday de los retos para estudiarla se origina en nuestra experiencia en el análisis de los usos, la apropiación y el control del espacio público y el estudio desde un enfoque antropológico de la relación que sostienen poblaciones precarizadas con la urbe en dos ciudades medias. ${ }^{1}$ En estas experiencias identificamos que en sus plazas principales, jardines, parques y mercados ${ }^{2}$ hay una pluralidad de actores, usos y actuaciones incluso discordantes. A simple vista, estos sitios se manifiestan como lugares de encuentro y alteridad (Licona, 2007b: 42). En ellos coinciden "casi todos" los habitantes de la ciudad; pero no están todos y es ahí donde queremos detenernos, en la población que no está presente, en los ausentes de la ciudad y sus espacios. Son actores urbanos que pertenecen a grupos poblacionales precarizados cuya relación con la urbe y sus lugares emblemáticos es mínimay, en ciertos casos, inexistente. Son habitantes de zonas urbanas periféricas; pero, también de áreas centrales, para quienes la ciudad no agrupa sus voces y no forma parte de sus referentes identitarios o de sociabilidad.

En nuestras experiencias de investigación en zonas precarizadas y polígonos de pobreza ${ }^{3}$ los habitantes entrevistados refirieron que su relación con los espacios públicos de la ciudad es prácticamente nula, las causas son diversas: falta de recursos económicos para trasladarse, inseguridad en el entorno que habitan que los obliga a replegarse en sus viviendas y limitar las salidas, sobre todo de las mujeres al dedicarse casi de manera exclusiva al cuidado de los hijos y tareas del hogar, algunos otros refirieron no tener ningún asunto que atender en los lugares mencionados:

No tiene uno a que salir, hace como casi diez años que no voy para allá [el centro de la ciudad] no tengo a que ir, al centro de salud voy aquí a la otra colonia y con mi familia también, ésos son mis lugares [Paty, nota de campo, 24 de mayo de 2019].

No salgo de la casa para nada, yo soy la que cuido a los niños [Lourdes, nota de campo, 26 de julio de 2019].

1 Las experiencias examinadas son la capital de San Luis Potosí y León, Guanajuato; esta última con información recopilada en trabajo de campo por investigadores y becarios del proyecto Calidad de Vida y Crecimiento Urbano. Nuevas Desigualdades en León, Guanajuato 2019, financiado por la Universidad de Guanajuato como parte de su programa de apoyo a la investigación.

2 Algunos de los trabajos pioneros, como los de Ernesto Licona (2007c) inauguraron el análisis del Centro Histórico de Puebla partiendo de la idea de que sus plazas podían considerarse "estandartes de lo público"; este autor, basado en las ideas de Isaac Joseph, Manuel Delgado y otros estudiosos del espacio público, propuso entenderlos como espacios de circulación remarcados con una importante carga simbólica, contenedores de una diversidad que hoy debatimos.

3 Para el ayuntamiento leonés un polígono de pobreza es una zona de la ciudad donde se concentra la población que cuenta con diferentes niveles de carencias de acuerdo con los criterios del Consejo Nacional de Evaluación de la Política de Desarrollo Social (Coneval) y del índice de marginación urbana que calcula el Consejo Nacional de Población (Conapo). Estos polígonos coinciden con los núcleos de asentamientos irregulares que se fueron aglutinando a lo largo de varias décadas y que hoy son reconocidos como tales. A nivel nacional, León es uno de los dos municipios del estado de Guanajuato que se encuentran dentro de los 20 con más pobreza extrema con 600145 personas. 
La verdad yo no salgo, por lo mismo de la inseguridad. Así que entre mi hermana y yo pusimos el puesto [de ropa usada] lo saco en la mañana y aquí estoy al pendiente de los niños [Rosa, nota de campo, 2 de agosto de 2019].

$\mathrm{Al}$ explorar qué actividades realizan en los espacios públicos de la ciudad los participantes de casi una veintena de entrevistas refirió algún acercamiento sólo por cuestiones laborales; en general, no hay relación y para casi todos los entrevistados estos espacios no figuran en sus referentes como habitantes de la ciudad. La relación de estos grupos es limitada, es un segmento de población que experimenta lo público y lo privado desde escenarios más homogéneos que diversos, cuyo estudio también nos exige retos metodológicos para su abordaje, en la medida en que se trabaja en contextos de riesgo y violencia (Maldonado Aranda, 2013; Hjorth Boisen, 2018). Las prácticas espaciales de estos habitantes nos revelaron que habitan espacios urbanos aislados física y simbólicamente y que enfrentan un conjunto de problemas asociados a la precariedad con realidades más o menos homogéneas. Su relación con la ciudad es limitada: no la conocen, no la viven, no la recorren y sólo algunos la experimentan de manera parcial bajo condiciones de subordinación: como empleados de bajo nivel, comerciantes ambulantes o autoempleándose en calles y avenidas para limpiar parabrisas de autos o pedir dinero a los transeúntes.

Si bien es cierto que el análisis de los modos de habitar la ciudad y el estudio de las desigualdades urbanas ha contemplado la exclusión y segregación explorando casos como el de la población indígena o habitantes de calle (Reyes, 2017; Ruiz, 2017), partiendo de la idea de que el espacio público es plural y estas poblaciones pueden exigir su derecho a la ciudad, también lo es que esa diversidad es parcial, pues en las ciudades hay grupos completamente ajenos a las dinámicas de los espacios públicos. Con lo anterior no desdeñamos los análisis que hasta ahora se han emprendido, por el contrario, esas exploraciones sobre los modos de habitar de los usuarios cotidianos y de los que luchan por usar el espacio público han permitido notar la ausencia de otras poblaciones que ni siquiera se atreven a pisar ese espacio disputado y desigual (Giglia, 2017: 14).

Las principales plazas, corredores, calles, edificios y otros espacios públicos le son ajenos a esta población que sólo conoce la realidad de las ínsulas inconexas que habita. Eso nos condujo a mirar de nuevo al espacio público, o lo que creíamos era tal cosa, desde su opacidad para reconocer que no es tan diverso, ni es tan público y que es difícil que el más acaudalado y el más pobre de una urbe estén bajo el mismo esquema de uso y apropiación. Asimismo nos colocó frente a un escenario que hizo explícita la dificultad de estudiar esos fragmentos de la vida social que parecen estar en tránsito continuo entre lo público y lo privado.

Para poder visibilizar y aproximarnos a las múltiples condiciones de la espacialidad urbana y su diversidad es necesario replantearnos cómo estamos abordándola, desde la academia requerimos conectar espacios desconectados en la realidad social, conformar equipos multidisciplinarios con ojos que simultáneamente vean cosas distintas, estos relatos múltiples de la alteridad pueden poner al descubierto actores no tradicionales, nuevos materialismos manifiestos en las prácticas corporales de los urbanitas, percibir afectos que abran ventanas de multiversos que resultan apremiantes en la reflexión sobre las ciudades (Reyna y Arce, 2015). Lo anterior no es algo nuevo, por el contrario, podríamos señalar que estas formas de mirar la diversidad urbana se nutren de los trabajos de investigación aplicada impulsados desde la década de 1990 en el campo de la salud en contextos agrícolas y otros proyectos inter-y transdisciplinarios (Taplin, Scheld y Low, 2002: 81) que son de gran utilidad para optimizar el análisis de la pluralidad en las ciudades desde sus propios ritmos, como es el caso de las etnografías rápidas propuestas para investigaciones con limitaciones de tiempo, recursos económicos y humanos, las cuales demandan ser examinadas desde otras disciplinas para validar la información y generar cuestionamientos sobre una realidad que sólo es posible comprender mediante trabajo en equipo, triangulación de métodos de investigación y un proceso operativo de interpretación de datos (Taplin, Scheld y Low, 2002: 92). Este diálogo y la combinación de técnicas y estrategias son imperiosos para alcanzar los resquicios inexplorados de la vida urbana.

El diálogo con otras disciplinas y la recuperación de estrategias no imaginadas nos permitirá quebrar cortezas teóricas y metodológicas para eludir la homogenización de ideas y lograr así la comprensión del espacio público desde su fragmentación. Hace falta reconocer que la diversidad que conocíamos no es tan heterogénea y que nuestra tarea más apremiante es encontrar las intersecciones y adaptar las herramientas indispensables que pueden ser la etnografía enfocada, los recorridos a la deriva, las observaciones colectivas y otras tantas que pueden surgir para lograr aproximarnos a los fragmentos dispersos de la diversidad contemporánea cuya dinámica nos obliga a concebir nuevos apuntalamientos para su abordaje. 


\section{Consideraciones finales}

Aquí hemos sostenido que la ciudad es un contenedor de la diversidad y que, en su búsqueda de la igualdad política y social, genera al mismo tiempo formas de homogeneización que difuminan la primera. Es decir, hay una dicotomía paradójica: incluir-excluir. ¿Cuál es el motor, entonces, que nos serviría para dar cuenta de la cohabitación del espacio público? Hemos propuesto que observar la ciudad desde una idea preconcebida de la cultura dará por resultado que la diversidad es promovida, respetada y fortalecida en sintonía con el proyecto liberal. Pero si nos detenemos a mirar con una perspectiva diferente, lo que aparece en el espacio público es una fragmentación de la misma, esto es, la construcción de manifestaciones insulares de la diversidad a través de campos semánticos o de zonificaciones que marcan reglas para el uso y la cohabitación del espacio público, de hecho, en la relación entre cada una de esas islas sociales hay una tensión constante que puede derivar en conflicto. Así, el uso del espacio público es un proceso constante de negociación entre quienes tienen acceso a él y crean, de manera inconsciente la mayor parte del tiempo, los islotes de interacción que procuran mantener un espacio vital entre ellos y, de este modo, evitar el conflicto.

Con todo y que existe un conjunto de normas formales que regulan el uso del espacio público, tal vez las más importantes son las informales, pues éstas se establecen y son administradas por los usuarios; $\mathrm{y}$, también, son respetadas por la autoridad. Con la mirada de siempre hemos de ver que el espacio y la cohabitación están organizados y hasta son predecibles. Con el lente que proponemos, lo que subyace es un constante proceso de exclusión social, pero igualmente de invisibilización. En este sentido, las categorías para la definición de la diversidad resultan insuficientes, pues ya no se trata de comprender la construcción de una hegemonía del concepto ciudadano, sino de entender que en el espacio público hay inclusiones y exclusiones que ocurren al mismo tiempo y de manera constante evitan el conflicto a través de una negociación del uso y la cohabitación (Viveiros de Castro, 2010: 24).

En este sentido, es preciso cambiar el prisma con el que se ve a la ciudad, a los fenómenos urbanos y al espacio público. La diversidad es reconocida en la medida en que el discurso de la inclusión funciona como bandera política. El resultado es una ciudad homogénea, en donde hay que cumplir con un conjunto de expectativas para no convertirse en el marginal, en la alteridad, en aquello que habita al margen y, por lo tanto, es excluido e invisibilizado. ¿Cuáles son las ca- racterísticas de esa homogeneidad? Variarán según el contexto social en el que se desarrolle, pero involucrarán tono de piel, forma de vestir, modo de expresarse, grado educativo, capacidad de consumo, por mencionar algunos; luego, esa homogeneidad será fragmentada, no como parte de lo diverso, sino como manifestaciones culturales de una misma población, es decir, no como formas de entenderse en este mundo, sino como construcciones sociales a partir del reconocimiento institucional de su diferencia. En otras palabras, la diversidad sólo existe en la medida en que se expresa en los espacios públicos en una fragmentación de las sociabilidades regulada por reglas formales o informales que son validadas por el actor institucional inmediato o mediato (Holbraad, cit. en González-Abrisketa y Carro-Ripalda, 2016: 114; Pedersen, 2011).

¿Quiere esto decir que todos aquellos que no cumplan con lo esperado son invisibilizados del espacio público? Quizá. Los espacios "abiertos a la sociedad" son en realidad sitios a los que pueden acceder grupos determinados por dos razones: el discurso institucional que establece las características fundamentales del sujeto-actor del espacio público, y las prácticas informales que pueden reforzar ese discurso o, bien, resistirse a él, pero que reproducen las exclusiones y segregaciones sociales construidas desde lo institucional. El éxito de esta forma de organización de la sociedad es que ha generado campos semánticos que llevan a los marginales a establecer que hay lugares vedados para ellos. Por ejemplo, como ya se ha sostenido en diversos estudios (Solís, 2011; Villagómez Ornelas, 2019), la pobreza no sólo es una condición económica, sino también un estatus social que sostiene dos discursos y acciones antagónicas: el reconocimiento de que los pobres deben ser incluidos en el desarrollo y arropados por la urbe que habitan, mientras que, a la vez, sistemáticamente se excluye del desarrollo a la población en esas condiciones. Aunque éste es el ejemplo extremo, también hay procesos de exclusión social entre quienes representan el modelo ideal del individuo citadino. Por ejemplo, sectores de la población deciden no asistir a los conciertos de la llamada música clásica porque se ha creado un campo semántico en torno a los conciertos de orquestas sinfónicas o filarmónicas: son espacios públicos a los que se debe ir vestido de una manera particular; se debe saber de música para disfrutar el concierto. Así que, al asistir a un concierto de este tipo, lo usual es encontrar gente que va vestida con un estilo formal, que bebe vino de mesa y que aparentemente sabe de música sinfónica, es decir, "es gente culta”. Mientras que el resto de la población, que se asume como "no 
culta" o "poco culta", prefiere mantenerse al margen, les ha sido vedado un espacio que debería ser público, pero que en realidad se convierte en un sitio para una élite (autoconstruida) de la cultura.

Pero, ¿cómo dar cuenta de ello? En el texto indicamos que es necesario utilizar de un modo diferente las herramientas metodológicas con las que se cuenta y generar, si es preciso, nuevas. Desde nuestro punto de vista, por ahora basta con cambiar la mirada y observar todo aquello que no está manifiesto, la lectura del afuera, como plantearía Foucault (1997). ¿Qué es lo observable y qué no logro asir con las taxonomías generadas hasta el momento? ¿Qué dice todo aquello que es manifiesto?

Es decir, sin perder de vista que se trata del estudio de seres humanos (De la Peña, 2015) y sus interacciones, lo que debemos tener presente en nuestras prácticas etnográficas en los entornos urbanos es que muchas de esas relaciones sociales son mediadas por prácticas institucionales (formales e informales) que sesgan la agencia de cada uno de los actores; en este sentido, lo que aquí planteamos es que ésta es dirigida por un conjunto de prácticas que fragmentan y crean aislamientos que mantienen una constante tensión unos con otros, esta organización insular de la sociedad también puede explicar detalles del desarrollo histórico-social de una población (Lacarrieu, 2007), ¿qué nos desvela eso?: la necesidad implícita de la generación de diálogos interdisciplinarios que combinen, por un lado, metodologías y, por otro, análisis que complementen lo que se observa desde los ejercicios etnográficos (Gershon, 2011).

\section{Fuentes}

Almada Flores, Hugo

2014 "La apropiación del espacio público a través de las prácticas deportivas juveniles”, tesis de doctorado, El Colegio de la Frontera Norte, Tijuana, $192 \mathrm{pp}$

Azuela, Antonio

2016 "Introducción. Una especie de neorrealismo jurídico", en Antonio Azuela (coord.), La ciudad y sus reglas. Sobre la huella del derecho en el orden urbano, Universidad Nacional Autónoma de México/Procuraduría Ambiental y del Ordenamiento Territorial de la Ciudad de

Balbi, Fernando México, México, pp. 9-40.

2017 "Comparación, etnografía y generalización", en Anuario Antropológico, vol. 42, núm. 1, pp. 9-35.

BanerJee, AbhiJit V.

Y ESTHER DUFLO

2019 Repensar la pobreza. Un giro radical en la lucha contra la desigualdad global, Taurus, México, 372 pp.
Chávez, Mónica

2014 Identidad étnica, migración y socialización urbana. Profesionistas indígenas de la Huasteca enlacapitalpotosina, CentrodeInvestigaciones y Estudios Superiores en Antropología Social/El Colegio de San Luis, México, 255 pp.

Delgado, Manuel

2008 El animal público, Anagrama, Barcelona, $224 \mathrm{pp}$

Del Mármol, Camila, Joan Frigolé

Y SUSANA NAROTZKY (COORDS.)

2010 Los lindes del patrimonio. Consumo y valores del pasado, Instituto Catalán de Antropología, Barcelona, $421 \mathrm{pp}$.

De la Peña, Guillermo

2015 "Ciudades, diversidades y ciudadanías en la antropología mexicana”, en Desacatos, núm. 48, pp. 177-191.

Durán Segura, Luis Armando

2011 "Miradas urbanas sobre el espacio público: el flâneur, la deriva y la etnografía de lo urbano", en Reflexiones, núm. 90, pp. 137-144.

Foucault, Michel

1997 El pensamiento del afuera, Pre-Textos, España, $40 \mathrm{pp}$.

García Canclini, Néstor

2005 La antropología urbana en México, Fondo de Cultura Económica/Universidad Autónoma Metropolitana, México, $381 \mathrm{pp}$.

Gershon, ILANA

2011 "Neoliberal agency", en Current Anthropology, vol. 52, núm. 4, pp. 537-555.

Giglia, Angela

2002 "Privatización del espacio, autosegregación y participación ciudadana en la Ciudad de México: el caso de las calles cerradas en la zona de Coapa (Tlalpan, Distrito Federal)", en Trace, Procesos Mexicanos y Centroamericanos, núm. 42 , pp. 71-78.

Giglia, Angela

2016 "Reglamentos y reglas de usos de la Alameda Central de la Ciudad de México: un régimen híbrido", en Antonio Azuela (coord.), La ciudad y sus reglas. Sobre la huella del derecho en el orden urbano, Universidad Nacional Autónoma de México/Procuraduría Ambiental y del Ordenamiento Territorial de la Ciudad de México, México, pp. 381-422.

Giglia, Angela

2017 "Espacios públicos, sociabilidad y orden urbano. Algunas reflexiones desde la Ciudad de México sobre el auge de las políticas de revitalización urbana”, en Cuestión Urbana, año 2 , núm. 2, pp. 15-28.

Giglia, Angela (COORD.)

2018 Renovación urbana, modos de habitar y desigualdad en la ciudad de México, Universidad Autónoma Metropolitana, México, 404 pp.

GonZÁlez-Abrisketa, Olatz

y Susana CARRo-Ripalda

2016 "La apertura ontológica de la antropología contemporánea", en Disparidades. Revista de Antropología, vol. 71, núm. 1, pp. 101-128.

GuédeZ, Víctor

2005 "La diversidad y la inclusión: implicaciones para la cultura y la educación”, en SAPIENS, vol. 6, núm. 1, pp. 107-132.

Hernández García, Jaime

2013 "Construcción social de espacio público en 
barrios populares de Bogotá", en Revista INVI, vol. 28, núm. 78 , pp. 143-180.

Huorth Boisen, Susann Vallentin

2018 "Evaluación y reducción de riesgo en el trabajo de campo", en Alteridades, vol. 28, núm. 56, pp. 73-84.

Hoffman, Odile y Abelardo Morales Gamboa

2018 "El territorio como recurso. Introducción al volumen", en Odile Hoffman y Abelardo Morales Gamboa (coords.), El territorio como recurso. Movilidad y apropiación del espacio en México y Centroamérica, Facultad Latinoamericana de Ciencias Sociales, San José de Costa Rica, pp. 11-22.

Jirón, PAOLA E IMILAN Walter

2016 "Observando juntos en movimiento: posibilidades, desafíos o encrucijadas de una etnografía colectiva", en Alteridades, vol. 26, núm. 52, pp. 51-64.

LACARRIEU, MóNICA

2007 "Una antropología de las ciudades y la ciudad de los antropólogos", en Nueva Antropología, vol. 20, núm. 67, pp. 14-39.

Leal Sorcia, Olivia

2012 "Experimentación y nuevos temas en la etnografía de grupos indígenas en ciudades mexicanas”, en Andamios, núm. 9, pp. 103-126.

Licona VAlencia, ERNESTO

2007a "Introducción", en Ernesto Licona Valencia (coord.), El zócalo de la ciudad de Puebla. Actores y apropiación social del espacio, Benemérita Universidad Autónoma de Puebla/ Universidad Autónoma Metropolitana, México, pp. 11-18.

Licona Valencia, ERnesto

2007b "Espacio y cultura: un acercamiento al espacio público", en Ernesto Licona Valencia (coord.), El zócalo de la ciudad de Puebla. Actores y apropiación social del espacio, Benemérita Universidad Autónoma de Puebla/ Universidad Autónoma Metropolitana, México, pp. 19-44.

Licona VAlencia, ERnesto (COORD.)

2007c El zócalo de la ciudad de Puebla. Actores y apropiación social del espacio, Benemérita Universidad Autónoma de Puebla/Universidad Autónoma Metropolitana, México, 117 pp.

List, MAuricio

2007a "Masculinidades urbanas. Una reflexión a partir de algunos ejemplos de la ciudad de Puebla”, en María Ana Portal (coord.), Espacios públicos y prácticas metropolitanas, Universidad Autónoma Metropolitana, México, pp. 177-206.

List, Mauricio

2007b "Hombres, espacio, socialidad", en Ernesto Licona Valencia (coord.), El zócalo de la ciudad de Puebla. Actores y apropiación social del espacio, Benemérita Universidad Autónoma de Puebla/Universidad Autónoma Metropolitana, México, pp. 119-144.

Maldonado Aranda, Salvador

2013 "Desafíos etnográficos en el estudio de la violencia. Experiencias de una investigación", en Avá. Revista de Antropología, núm. 22, pp. 123-144.

Melé, Patrice

2016 "Incertidumbres y regulaciones urbanas: el papel de la calificación jurídica del espacio", en Antonio Azuela (coord.), La ciudad y sus reglas. Sobre la huella del derecho en el orden urbano, Universidad Nacional Autónoma de México/Procuraduría Ambiental y del Ordenamiento Territorial de la Ciudad de México, México, pp. 43-82.

Mier y Terán, Arturo, Isabel Vázguez

Y Alicia ZicCARDI

2012 "Pobreza urbana, segregación residencial y mejoramiento del espacio público en la Ciudad de México", en Sociologías, vol. 14, núm. 30, pp. 118-155.

MONNET, JÉRÔME

1996 "Espacio público, comercio y urbanidad en Francia, México y Estados Unidos", en Alteridades, vol. 6, núm. 11, pp. 11-25.

Montes de Oca Barrera, Laura Beatriz

2015 "Entre activistas, funcionarios e industriales: Aplicación de la etnografía -enfocada y política- en escenarios de gobernanza", en Nueva antropología, vol. 28, núm. 83, pp. 25-46.

Nivón, EDUARDO

2005 "Hacia una antropología de las periferias urbanas”, en Néstor García Canclini (coord.), La antropología urbana en México, Fondo de Cultura Económica/Universidad Autónoma Metropolitana, México, pp. 140-163.

Portal, María Ana

2007 "Introducción, espacio público y transformaciones urbanas en espacios públicos y prácticas metropolitanas”, en María Ana Portal (coord.), Espacios públicos y prácticas metropolitanas, Universidad Autónoma Metropolitana, México, pp. 45-68.

Restrepo, EduARDo

2012 Antropología y estudios culturales: disputas y confluencias desde la periferia, Siglo xxI, Buenos Aires, 240 pp.

Reyes, Alejandro

2017 "Modos de habitar la colonia Roma: el uso del espacio público por jóvenes residentes en vecindades otomíes”, en Angela Giglia (coord.), Renovación urbana, modos de habitar y desigualdad en la Ciudad de México, Universidad Autónoma Metropolitana/Juan Pablos Editor, México, pp. 219-250.

Reyna, Oscar y Alberto Arce

2015 "Cosmopolítica, patrimonio y contradesarrollo: la modificación de espacios de acción actoral ante el riesgo de la explotación minera en el Sitio Sagrado Natural de Wirikuta", en Moisés Gámez (coord.), Minería y capital transnacional sobre un territorio en riesgo. Análisis interdisciplinario sobre el sitio sagrado natural de Wirikuta, El Colegio de San Luis, México, pp. 93-123.

RoJas, MaURICIO

2007 "Hacia nuevas configuraciones de lo público y lo privado en espacios urbanos", en María Ana Portal (coord.), Espacios públicos y prácticas metropolitanas, Universidad Autónoma Metropolitana, México, pp. 69-110.

Rosanvallon, PierRe

2010 La legitimidad democrática. Imparcialidad, reflexividad y proximidad, Paidós, Barcelona, $317 \mathrm{pp}$.

Rosas Mantecón, Ana

2005 "Las disputas por el patrimonio. Transformaciones analíticas y contextuales de la 
problemática patrimonial en México", en Néstor García Canclini (coord.), La antropología urbana en México, Fondo de Cultura Económica/ Universidad Autónoma Metropolitana, México, pp. 60-88.

Ruiz, Alí 2017

Sevilla, Amparo

"Criminalización de la vida en calle en la Ciudad de México", en Angela Giglia (coord.), Renovación urbana, modos de habitar y desigualdad en la Ciudad de México, Universidad Autónoma Metropolitana/Juan Pablos Editor, México, pp. 321-352.

\section{y María Ana Portal}

2005 "Las fiestas en el ámbito urbano", en Néstor García Canclini (coord.), La antropología urbana en México, Fondo de Cultura Económica/ Universidad Autónoma Metropolitana, Méxi-

SOLÍs, PATRICIO co, pp. 341-372.

2011 "Desigualdad y movilidad social en la Ciudad de México", en Estudios Sociológicos, vol. 29, núm. 85, pp. 283-298.

Taplin, Dana H., Suzanne Scheld

y Setha M. Low

2002 "Rapid ethnographic assessment in urban parks: A case of study of Independence National Historical Park", en Human Organization, vol. 61, núm. 1, pp. 80-93.

TÉLlEZ, LEÓN

2018 "Renovación urbana, nostalgia y habitar en el Centro de la ciudad de México", en Angela Giglia (coord.), Renovación urbana, modos de habitar y desigualdad en la ciudad de México, Universidad Autónoma Metropolitana, México, pp. 183-216.

Urteaga Castro-Pozo, Maritza

2007 "Usos y apropiaciones del zócalo por jóvenes", en Ernesto Licona Valencia (coord.), El zócalo de la ciudad de Puebla. Actores y apropiación social del espacio, Benemérita Universidad Autónoma de Puebla/Universidad Autónoma Metropolitana, México, pp. 81-117.

Urteaga Castro-Pozo, Maritza

E Inés Cornejo Portugal

1995 "La privatización afectiva de los espacios comerciales por las y los jóvenes”, en Ciudades, núm. 27, pp. 24-28.

Urteaga Castro-Pozo, Maritza

y CARLOS FEIXA

2005 "De jóvenes, músicas y las dificultades de integrarse", en Néstor García Canclini (coord.), La antropología urbana en México, Fondo de Cultura Económica/Universidad Autónoma Metropolitana, México, pp. 265-298.

Valenzuela Arce, José Manuel

1988 ¡A la brava ése!, El Colegio de la Frontera Norte, México, 233 pp.

Vergara, Abilio

2005 "Introducción. El lugar antropológico", en Miguel Ángel Aguilar, Amparo Sevilla y Abilio Vergara (coords.), La ciudad desde sus lugares, trece ventanas etnográficas para una metrópoli, Porrúa/Consejo Nacional para la Cultura y las Artes/Universidad Autónoma Metropolitana, México, pp. 5-33.

Villagómez Ornelas, Paloma

2019 "La constitución social de experiencias alimentarias en la pobreza. Un estudio cualitativo con familias de estratos populares en Iztapalapa, Ciudad de México", tesis de doctorado, El Colegio de México, México, $391 \mathrm{pp}$.

Viveiros de Castro, Eduardo

2010 Metafísicas caníbales: líneas de antropología postestructural, Katz Editores, Buenos Aires, $258 \mathrm{pp}$. 